\title{
Quantum spin Hall phase in multilayer graphene
}

\author{
N. A. García-Martínez, J. L. Lado, and J. Fernández-Rossier* \\ International Iberian Nanotechnology Laboratory (INL), Avenida Mestre José Veiga, 4715-330 Braga, Portugal
}

(Received 26 March 2015; revised manuscript received 22 May 2015; published 30 June 2015)

\begin{abstract}
The so-called quantum spin Hall phase is a topologically nontrivial insulating phase that is predicted to appear in graphene and graphenelike systems. In this paper we address the question of whether this topological property persists in multilayered systems. We consider two situations: purely multilayer graphene and heterostructures where graphene is encapsulated by trivial insulators with a strong spin-orbit coupling. We use a four-orbital tight-binding model that includes full atomic spin-orbit coupling and we calculate the $Z_{2}$ topological invariant of the bulk states as well as the edge states of semi-infinite crystals with armchair termination. For homogeneous multilayers we find that even when the spin-orbit interaction opens a gap for all possible stackings, only those with an odd number of layers host gapless edge states while those with an even number of layers are trivial insulators. For heterostructures where graphene is encapsulated by trivial insulators, it turns out that interlayer coupling is able to induce a topological gap whose size is controlled by the spin-orbit coupling of the encapsulating materials, indicating that the quantum spin Hall phase can be induced by proximity to trivial insulators.
\end{abstract}

\section{INTRODUCTION}

In their seminal papers [1], Kane and Mele established the existence of two fundamentally different types of band insulators with time reversal symmetry in two dimensions (2D), dubbed trivial and topological. Remarkably, it was predicted that monolayer graphene would be topological, giving rise to protected chiral gapless edge states. Importantly, this opened a new venue in condensed matter physics, the quest for searching and designing topological states in twodimensional systems.

The nature of the topological state in graphene comes from intrinsic spin-orbit coupling (SOC). In particular, SOC will open gaps of opposite signs at the two Dirac points, in contrast to a trivial gap where a staggered potential opens in the honeycomb lattice, with the same sign at the two valleys. This twisting of wave functions in reciprocal space leads to the appearance of in-gap states at the boundaries of the material. Subsequent work [2-4] found that the size of the SOC gap in graphene was very small, and attention was shifted to other systems, such as $\mathrm{CdTe} / \mathrm{HgTe}$ quantum wells [5], in which the quantum spin Hall (QSH) phase was found [6], as well as to bulk systems, for which the notion of topologically nontrivial insulators was extended. Experimental evidence for the quantum spin Hall phase has also been found in other systems, such as $\mathrm{Bi}(111)$ atomically thin layers $[7,8]$ and $\mathrm{InSb} / \mathrm{GaSb}$ quantum wells $[9,10]$.

Multilayers of two-dimensional materials are also potential candidates to sustain topological states. In particular, their appeal comes from the tunability of stacking different numbers of layers, or even different materials. In the present work we will focus on the study of a particular type of multilayer system, whose basic building blocks are graphenelike systems. We will study mainly two families of multilayers. First, we consider multilayered systems formed by graphenelike insulators using the SOC as a free parameter, so the main concepts should

\footnotetext{
* On leave from Departamento de Física Aplicada, Universidad de Alicante, Alicante, Spain.
}

be suitable for systems such as graphene, silicene [11-13], germanene [14], or stanene [15] (in fact, our methods make it easy to extended this kind of analysis to the case of bismuth [7,8,16] and metal-organic frameworks [17-23]). Second, stacks are formed by a layer of graphene encapsulated by some trivial insulator with a strong SOC.

From a practical point of view, several reasons motivate this work. First, there is a generic interest in the possibility of engineering the electronic properties of two-dimensional crystals, such as graphene, $h$-BN, and transition metal dichalcogenides, by combining them into multilayers [24-26]. Stacking monolayers of the same type is also a very interesting and widely studied possibility.

Our second motivation is to study the behavior of the topological gap as we increase the number of layers in the system. In the case of graphene, it is well known that key electronic properties, such as the pattern of Landau levels and the density of states at the Dirac point, are drastically modified for bilayer [27] and trilayer graphene [28-32]. Recent experimental work shows that some sort of magnetic order can occur, even at $B=0$ in bilayers [33,34], trilayers [35], and even tetralayers [36]. These last trivial symmetry breaking states will compete with the potential topological states studied in our work.

A third motivation comes from recent experiments [37] that report a very large enhancement of the spin Hall effect for graphene deposited on top of $\mathrm{WS}_{2}$, as a trivial semiconductor with a quite large SOC. This inspires the calculation for graphene placed between two insulators with a trivial band gap, large SOC, and broken inversion symmetry, to mimic the properties of $\mathrm{WS}_{2}$ and related transition metal dichalcogenides.

Furthermore, we have also a formal motivation. It is not obvious a priori that the original second-neighbor hopping Hamiltonian [1] can be applied to multilayer graphene [38]. In monolayer graphene the $p_{z}$ orbitals are strictly decoupled from the $s, p_{x}, p_{y}$ orbitals, due to mirror symmetry with respect to the plane. In the monolayer, SOC mixes $p_{z}$ with $p_{x}$ and $p_{y}$ orbitals of opposite spin and, when treated perturbatively, leads to an effective Hamiltonian [1] with a spin-dependent effective second-neighbor hopping between $p_{z}$ orbitals that conserves $S_{z}$. In multilayer graphene this is no longer true, since electrons 
in a $p_{z}$ orbital in one layer can hop to the $s$ orbital of atoms in the next layer. When SOC is added to the model, we expect that this $s-p_{z}$ mixing naturally leads to spin-mixing terms in the Hamiltonian, which is indeed the case [39]. The presence of this spin-flip channel interaction casts doubt on the validity of the spin-conserving Kane-Mele model for multilayers [40,41] and motivates our choice of the standard [2,42-46] four-orbital tight-binding calculations.

The rest of this work is organized as follows. In Sec. II we briefly review the tight-binding model and the procedures to determine the existence of a QSH phase applied to the homogeneous case, studying the relation between the interlayer coupling and the topological properties of the system. In Sec. III the same methodology is applied to the case of a heterogeneous structure, graphene encapsulated by a trivial insulator, finding that topological properties can be induced even by trivial neighboring layers. Finally, in Sec. IV we summarize our findings.

\section{HOMOGENEOUS MULTILAYERS}

Monolayer graphene consists of a triangular lattice with two atoms per unit cell that leads, in reciprocal space, to a hexagonal Brillouin zone that hosts Dirac cones in its corners. When $N$ layers are considered, the crystalline structure remains the same, only there will be $2 N$ atoms per unit cell. We shall only use the so-called Bernal stacking, shown in Fig. 1, which is the ground state configuration, according to both density functional theory (DFT) calculations and experimental evidence [47-49]. In Bernal stacked materials an atom from the sublattice $B(A)$ sits on top of an atom belonging to the other sublattice $A(B)$. For $N=2$ there is only one way to achieve this, but for $N>2$ there are different possible stacking orders. In Fig. 1 we show the different possibilities for $N \leqslant 4$, with a self-evident notation.

\section{A. Model}

We describe the multilayers with the following tightbinding Hamiltonian,

$$
H=H_{\mathrm{ML}}+\eta H_{\mathrm{inter}}+\lambda \vec{L} \cdot \vec{S}
$$

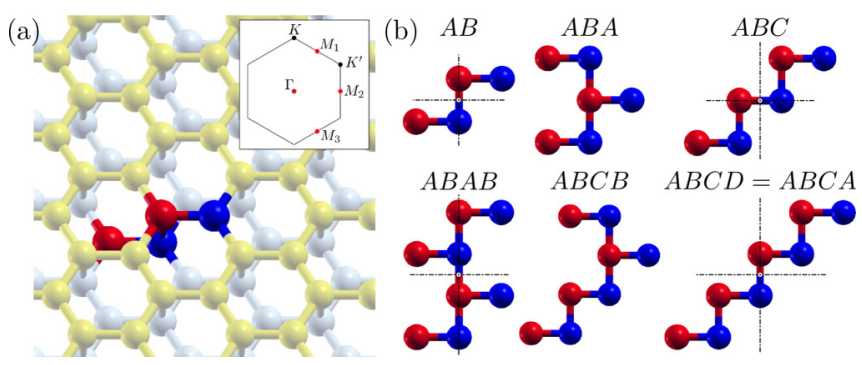

FIG. 1. (Color online) (a) Crystal structure of a bilayer graphene system with a highlighted unit cell. Different colors for each layer are used to distinguish the two layers. In the inset the first Brillouin zone is depicted with the high symmetry points and the time reversal invariant momenta colored in red. (b) Side view of the unit cells for all the different stackings studied. For stackings with inversion symmetry, the inversion center is shown at the crossing point of the dashed lines. For both panels, red and blue denote sublattices. where $H_{\mathrm{ML}}$ and $H_{\text {inter }}$ account for the intralayer and interlayer hoppings, respectively, and the last term is the intra-atomic SOC. Our tight-binding model is based on four atomic orbitals, $s, p_{x}, p_{y}$, and $p_{z}$. Both the intralayer and interlayer hoppings are described within the Slater-Koster formalism [50]. The intralayer hopping parameters are taken from Ref. [51]. In order to study the effect of interlayer coupling, the interlayer terms are scaled by a dimensionless parameter $\eta$. When $\eta=1$, the ratio between interlayer and intralayer $V_{p p \pi}$ in graphene is taken as [52] 0.13. Unless otherwise stated, in all our calculations we have $\eta=1$. Within this model, the dimension of the Hilbert space for the minimal unit cell of a crystal with $N$ layers is $4 \times 2 \times 2 \times N=16 N$ (four orbitals per atom, two atoms per layer, plus two possible spin orientations).

Without SOC, this model reproduces the very well known band structure of graphene $(N=1)$ and multilayer graphene $N>1$, which portrays these systems as zero-gap semiconductors. Within this model, SOC is known to open a gap in the monolayer [2] as well as in the bilayer [29,38,39]. In the case of monolayer graphene, the gap is known to be topological. Within this model, the computed value of the gap $1.46 \mu \mathrm{eV}$ when we take a realistic value of the atomic spin-orbit coupling, $\lambda=10 \mathrm{meV}$. This gap is much smaller than the ones obtained with accurate density functional theory (DFT) calculations, in the range of $30 \mu \mathrm{eV}$ [29]. The reason for the discrepancy turns out to be that the major contribution to the SOC gap at the Dirac point comes from the coupling to the higher energy $d$ bands [29]. The latter is a simple consequence of the fact that SOC opens a gap in second order in the coupling in the Dirac points when projected over the $p$ band. In comparison, SOC acts as first order when considering channels involving the $d$ band. Nevertheless, interlayer hopping may open a first-order spin-flipping channel in the $p$ manifold, becoming of the same order as the intrinsic spin-conserving $d$-level contribution. These last processes would be the ones missing in the multilayer Kane-Mele model, and should be added for completeness. In our case, for the sake of simplicity, we will focus on the spin-flipping channel, and use a four-orbital tight-binding model considering $\lambda$ as a free parameter. Future work shall focus on the effect of the $d$ levels in multilayer graphene, which will not be addressed here.

The effect of SOC on the band structure of the multilayers can be summarized by the following points:

1. SOC opens up a gap for all the $N$ stacked layers considered, reproducing the existing results [39] for the case of $N=2$. Notice that in the case of $A B A$ and $A B C B$ stackings, the system remains gapless up to a critical value of $\lambda$. This peculiarity is related to the nonuniform evolution of the SO splitting of the linear and nonlinear bands, as shown in Fig. 2.

2 . The scaling of the gap with $\lambda$ is very similar for the monolayer and $N=2,3,4$ multilayers, as shown in Fig. 2. Therefore, it is expected that, within this model, the gap opened by the intrinsic SOC might be as small in multilayers as it is in monolayers.

3. The magnitude of the band gap is insensitive to interlayer coupling. This result is somewhat surprising, since, together with atomic SOC, the interlayer coupling opens a spin-flip channel that is otherwise missing in the monolayer case. For the $A B$ bilayer, this can be understood considering the Hamiltonian at the Dirac point, for a given spin flavor. In the 

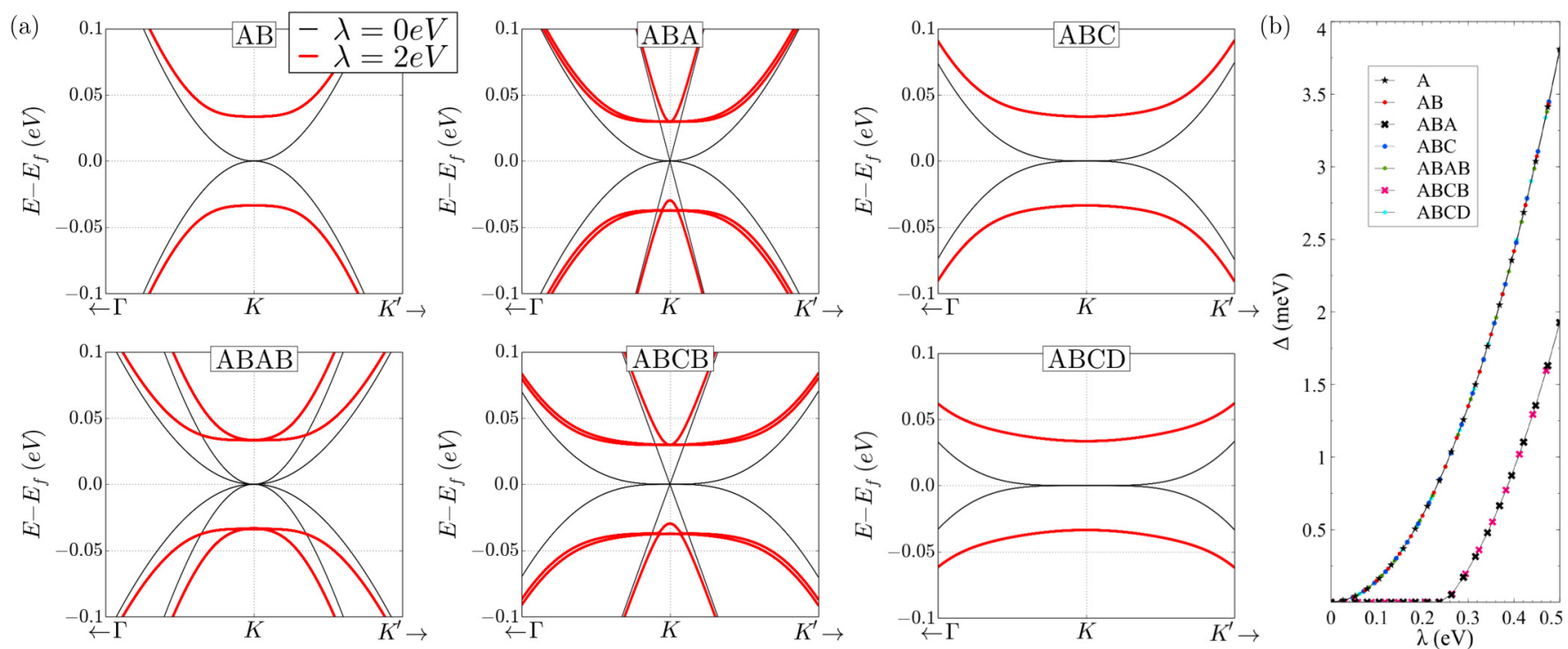

FIG. 2. (Color online) In (a) the band structure close to the $K$ point is shown for all possible stackings of multilayer graphene with $N=2,3,4$. Only when $\lambda \neq 0$ (red line) is a gap opened at the Dirac points. Note that for $A B A$ and $A B C B$ stackings there are linear bands when $\lambda=0$ that, when the SOC is switched on, cause a smaller gap than in the other cases. In (b) the dependence of the gap with the SOC $\lambda$ is shown. The anomalous behavior for the $A B A$ and $A B C B$ stackings is just due to the linear bands mentioned before.

basis $\left\{A_{1}, B_{1}, A_{2}, B_{2}\right\}$ the low energy Hamiltonian is given by

$$
H(K)=\left(\begin{array}{cccc}
\Delta / 2 & 0 & 0 & 0 \\
0 & -\Delta / 2 & \eta t & 0 \\
0 & \eta t^{\dagger} & \Delta / 2 & 0 \\
0 & 0 & 0 & -\Delta / 2
\end{array}\right),
$$

where $\Delta$ is the SO gap. When $\eta=0$, this Hamiltonian represents two decoupled monolayers. For finite $\eta$ the eigenvalues are $E_{ \pm}^{1}= \pm \Delta / 2$ and $E_{ \pm}^{2}= \pm \sqrt{(\eta t)^{2}+(\Delta / 2)^{2}}$. Since $\eta t \gg$ $\Delta$, the gap is still controlled by the $E^{1}$ couple and is thereby identical to that of the decoupled monolayers. As a result, switching on the interlayer coupling does not close the SOC gap of the monolayer, as shown in Fig. 2. As a consequence, the ground state of two decoupled $(\eta=0)$ monolayers can be adiabatically connected to the ground state of the bilayer $(\eta=1)$.

The last observation leads to the following result: Odd $N$ stacked graphene will be quantum spin Hall insulators (QSHIs), whereas even $N$ will not. More precisely, for a system of $N$ decoupled monolayers, the $Z_{2}$ invariant is

$$
Z_{2}(N)=\left[Z_{2}(1)\right]^{N} \text {. }
$$

Since the gap opened by $\lambda$ remains unaffected when switching on the interlayer coupling $\eta$, the value of $Z_{2}$ for graphenelike multilayers is also given by Eq. (3). We have verified that this qualitative behavior remains unchanged when $d$ channels are included in the picture by performing all-electron DFT calculations [53]. In the realistic low $\lambda$ limit, the gap is controlled by the linear contribution in $\lambda$, namely, the $d$ channel. For large SO, the $p$ channel that is quadratic in $\lambda$ becomes dominant, which corresponds to the situation in which the gap opening is properly captured by the SlaterKoster model. Thus, by artificially increasing $\lambda$ in a DFT calculation, it is possible to move from a $d$-dominated to a $p$-dominated SO gap. We have obtained that both limits are adiabatically connected without the gap closing, so that the topological properties in the low $\lambda$ limit are the same as in the large $\lambda$ limit.

In the following we verify Eq. (3) by using two different strategies. In the case of inversion symmetric structures, we compute the $Z_{2}$ invariant. In all cases, we compute the edge states and check whether or not they fill the gap. Independently of how the topological character is obtained, Eq. (3) holds in all the cases.

\section{B. Calculation of the $Z_{2}$ invariant}

Using the method developed by Fu and Kane in 2007 [54] for systems with inversion symmetry, it is possible to determine easily its topological character (the $Z_{2}$ invariant) by calculating the parity of the occupied Bloch wave functions at the time reversal invariant momenta (TRIMs),

$$
\delta_{i}=\prod_{m=1}^{N} \xi_{2 m}\left(\Gamma_{i}\right), \quad(-1)^{v}=\prod_{i} \delta_{i},
$$

where $\xi_{2 m}$ is the parity eigenvalue of the $2 m$ th occupied state at the TRIM $\Gamma_{i}=\left\{\Gamma, M_{1}, M_{2}, M_{3}\right\}$. Using this method, the topological character of a system will be determined just by the quantity $(-1)^{v}$, resulting in $(-1)^{v}=+1$ meaning trivial topology and $(-1)^{v}=-1$ meaning nontrivial topology. The calculation for the systems with inversion symmetry yields the following results:

\begin{tabular}{l|ccccc} 
& $A$ & $A B$ & $A B C$ & $A B A B$ & $A B C D$ \\
\hline$M_{1}$ & + & + & + & + & + \\
$M_{2}$ & + & + & + & + & + \\
$M_{3}$ & + & + & + & + & + \\
$\Gamma$ & - & + & - & + & + \\
\hline$(-1)^{v}$ & - & + & - & + & +
\end{tabular}



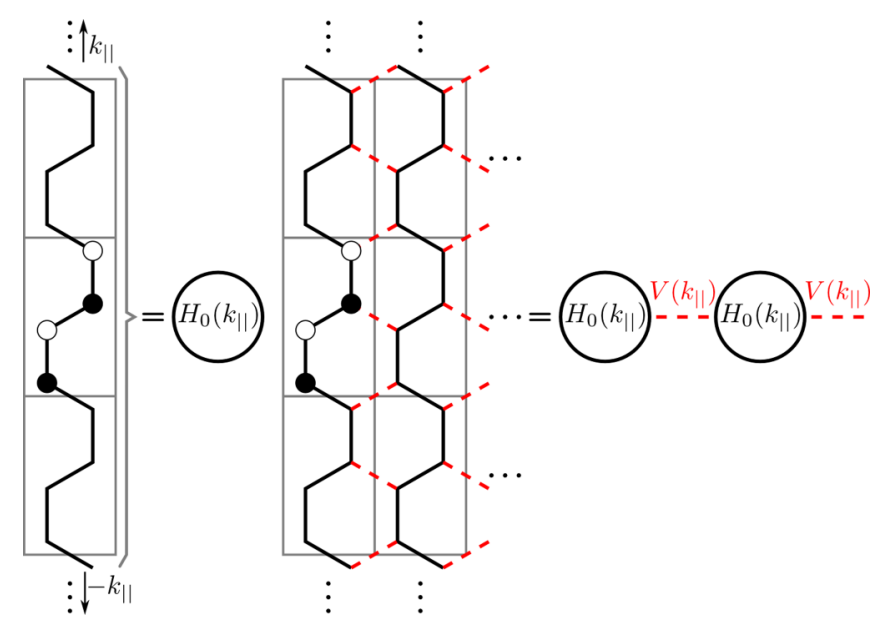

FIG. 3. (Color online) Scheme of the mapping between a semiinfinite crystal and a semi-infinite chain. The coupling between each linear chain (with $k_{\|}$well defined) is introduced by means of a selfenergy $\Sigma_{R}$.

This guarantees that $A$ and $A B C$ crystals are topological but the bilayers and tetralayers (with inversion symmetry) are not. For systems without inversion symmetry, the calculation of the $Z_{2}$ invariant requires a different approach [55-57] that requires a line integral of the Berry curvature over a contour in the Brillouin zone. Instead, we compute the edge states for these systems and invoke the bulk-edge correspondence to address the topological nature of those systems.

\section{Edge states}

To confirm Eq. (3) even for systems without inversion symmetry, we look for the presence of gapless edge states. We consider armchair-terminated semi-infinite crystals. Using translation invariance along the direction parallel to the edge, we block-diagonalize the Hamiltonian of the semi-infinite 2D crystal in terms of a collection of $k_{\|}$-dependent semi-infinite one-dimensional (1D) Hamiltonians, as indicated in Fig. 3. The 1D Hamiltonian describes unit cells with $4 N$ atoms, where $N$ stands for the number of graphene layers. The intracell terms are denoted by $H_{0}\left(k_{\|}\right)$and the intercell hoppings by $V\left(k_{\|}\right)$.

The surface Green's function of this block-tridiagonal semiinfinite matrix can be written as

$$
G^{\text {edge }}\left(E, k_{\|}\right)=\left[E+i \epsilon-H_{0}\left(k_{\|}\right)-\Sigma_{R}\left(k_{\|}\right)-\Sigma_{H}\left(k_{\|}\right)\right]^{-1},
$$

where $\Sigma_{R}\left(k_{\|}\right)$is a self-energy that accounts for the coupling to the semi-infinite crystal, $\Sigma_{H}\left(k_{\|}\right)$is the self-energy due to its interaction with the $H$ atoms included to get rid of the dangling bonds, and $\epsilon$ is a small analytic continuation.

The self-energy $\Sigma_{R}$ can be calculated employing a recursive Green's function method that leads to the following coupled
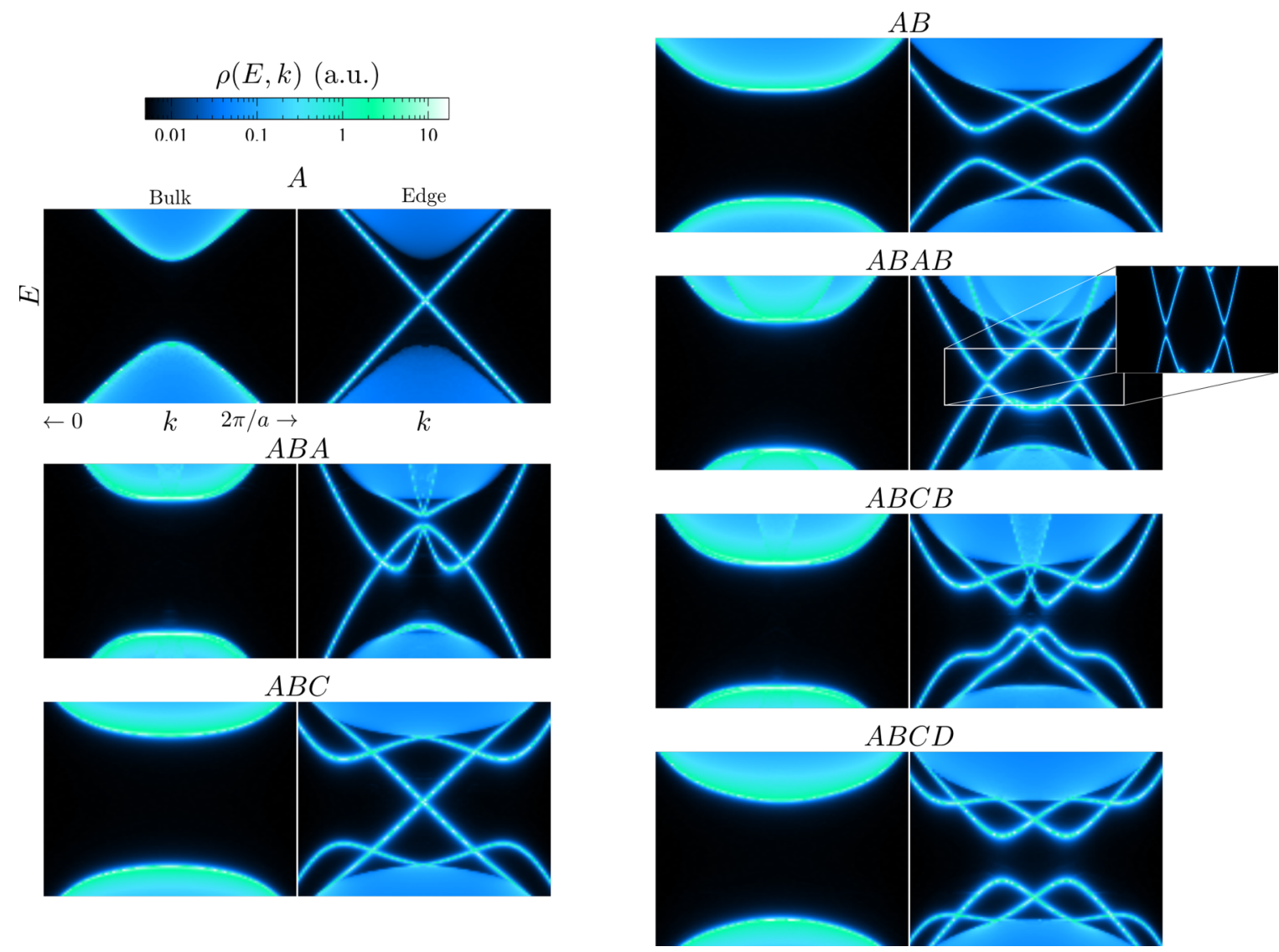

FIG. 4. (Color online) For each structure, the bulk and edge density of states (left and right panel, respectively). Gapless edge states appear only when an odd number of layers is considered independently of the stacking used. 
equations:

$$
\begin{aligned}
\Sigma_{R}\left(E, k_{\|}\right) & =V_{R}\left(k_{\|}\right) g_{R}\left(E, k_{\|}\right) V_{R}^{\dagger}\left(k_{\|}\right), \\
g_{R}\left(E, k_{\|}\right) & =\left[E-H_{0}\left(k_{\|}\right)-\Sigma_{R}\left(E, k_{\|}\right)\right]^{-1} .
\end{aligned}
$$

The $\Sigma_{H}\left(k_{\|}\right)$is calculated just as an additional iteration to the self-consistent calculation with the appropriate value for the hoppings $C-H$.

For a given $k_{\|}$we compute the density of states using

$$
\rho\left(E, k_{\|}\right)=-\frac{1}{\pi} \operatorname{Im}\left[G^{\text {edge }}\left(E, k_{\|}\right)\right] .
$$

Using a similar approach, we can also obtain the bulk density of states by calculating the bulk Green's function by recursion.

In Fig. 4 we show the density of states for both bulk and edge for all the stackings as a contour plot in the $k_{\|}, E$ plane. For each stacking the left panel shows the bulk density of states, which are gapped for all the stackings and the right panel shows the edge states. The calculations are done for a rather large value of $\lambda=2 \mathrm{eV}$. The first thing to notice is that, for such large values of $\lambda$, all the structures have edge states. However, only in the case of odd $N$, shown in the left column, the in-gap states are gapless. This is a necessary condition in order to have a QSHI. In contrast, all systems with even $N$ have edge states with a gap. Thereby, they are definitely not in the QSH phase, validating Eq. (3). Therefore, we conclude that odd $N$ graphene stacks are QSHIs and even $N$ are trivial insulators. In all cases, the gap opened by SOC is quite small.

\section{HETEROGENEOUS MULTILAYERS}

In the previous section we have seen that for homogeneous multilayers the gap opened by SOC has the same magnitude as that for the monolayer. Thereby, homogeneous multilayers of graphene would not improve the prospects for the observation of the QSH phase compared to the monolayer. We thus explore the case of a heterogeneous multilayer. This is motivated in part by recent experiments [37] that seem to indicate an enhancement of the SOC interaction in graphene due to proximity to $\mathrm{WS}_{2}$, a trivial semiconductor with quite large SOC and no inversion symmetry. There has also been plenty of work studying the enhancement of the SOC interaction in graphene due to proximity to heavy metals [58]. However, it would be much more interesting if graphene could be driven into a QSH phase by proximity to an insulator, so that the only conducting channels would be only at the edges of graphene.

Density functional calculations show [59] that a topological band gap opens in graphene on top of both $\mathrm{WS}_{2}$ and $\mathrm{WSe}_{2}$, two widely studied two-dimensional transition metal dichalcogenides (TMDs). The magnitude of this gap is in the range of a few meV, i.e., two or three orders of magnitude larger than the intrinsic SOC gap.

Here we propose a toy model to understand the opening of a nontrivial gap due to proximity to a trivial insulator with strong spin-orbit coupling. For that matter, we take graphene encapsulated between two monolayers of a trivial semiconductor with strong SOC and broken inversion symmetry. Specifically, the structure of these adjacent monolayers is that of a BN-like crystal [see Fig. 5(a)]. The choice of stacking is such that, (a)

(b)
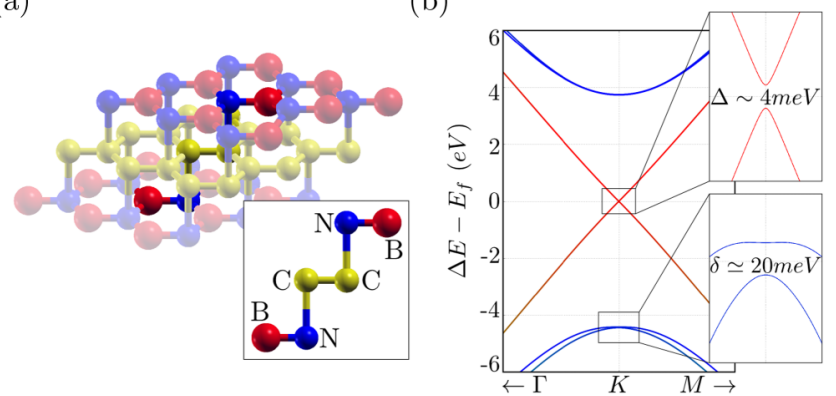

(c)

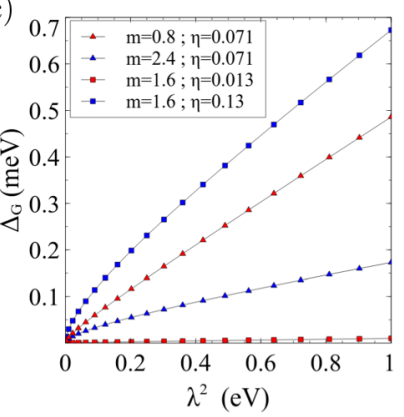

(d)

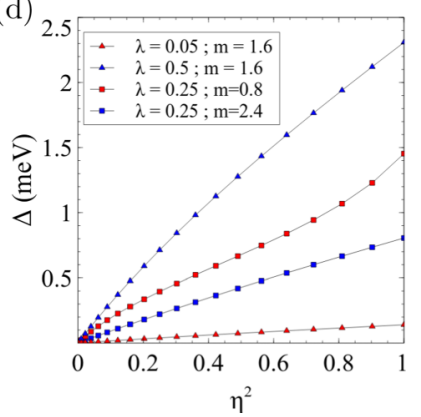

FIG. 5. (Color online) (a) shows the structure of the heterostructure considered. (b)-(d) show the dependence of the induced gap in graphene due to the proximity of the encapsulating layers. In (b) it can be seen that the gap is proportional to $\lambda^{2}$, and this estimation gets better as the gap of the insulating layers increases. (b) shows how the interlayer coupling $\eta$ produces the expected effect, since for a small interlayer coupling the induced gap is small but it grows quickly as $\eta$ increases. (c) shows the dependence of the induced gap with a sublattice imbalance.

globally, the structure has inversion symmetry. Otherwise, a trivial band gap would be opened by proximity [24].

The BN-like crystal is described with the same interatomic Slater-Koster parameters as graphene, but very different onsite parameters. In particular, we assume a large SOC $\lambda$ and a staggered potential $\pm m$ that breaks the inversion symmetry of the top and bottom layers. Since we are interested in the proximity effect, we turn off the atomic SOC of the graphene layer. As in the case of the homogeneous multilayers, the interlayer coupling is characterized by the dimensionless parameter $\eta$. In this case we impose zero SOC for the graphene layer, in order to study the proximity effect. For $\eta=0$ the bands of this system would be the superposition of those of the top and bottom insulators, with gap $2 m$, and the bands of graphene, whose Dirac cones would lie inside the gap. Broadly speaking, this picture remains the same as when the interlayer coupling is turned on. Interestingly, a nontrivial gap $\Delta$ opens in the Dirac cones only when $\eta \neq 0$ and $\lambda \neq 0$. We have verified that this gap satisfies the scaling

$$
\Delta \propto \frac{\lambda \eta^{2}}{m^{2}}
$$

in the limit of small $\lambda, \eta$, and $m^{-1}$. This result implies that graphene can borrow SOC from a neighboring trivial insulator layer via interlayer coupling. Using the method of TRIM we have verified that this insulator has $Z_{2}=(-1)^{v}=-1$, and is therefore topologically nontrivial. 
The magnitude of the proximity effect away from the weak coupling limit of Eq. (8) is shown in Fig. 5. We study the dependence of the proximity gap $\Delta$ as a function of both the SOC $\lambda$ and the interlayer coupling $\eta$ for two values of the encapsulating layer staggered potential $m$. It is apparent that, taking $m=2.0 \mathrm{eV}$ (a trivial gap $\sim 1.5 \mathrm{eV}$ ) and $\lambda \simeq$ $0.25 \mathrm{eV}$, values in line with those of 2D TMDs, the proximity gap is in the order of $1 \mathrm{meV}$, similar to the DFT results. Therefore, our model provides a reasonable justification of the DFT computations, which are certainly more complete.

Our toy model does not capture some probably important features of real heterogeneous multilayers. For instance, the interlayer interaction could break inversion symmetry which is expected to open a trivial gap. In addition, the geometry of our encapsulating layers was chosen to minimize the size of the unit cell, rather than to describe a real material. In general, the coupling of graphene to other 2D crystals will imply a new length scale, given by the size of the new unit cell. In this setup, the inversion symmetry breaking could average out.

\section{CONCLUSIONS}

We have studied the quantum spin Hall phase in multilayer graphene and in graphene encapsulated by a trivial semicon- ductor. In the case of multilayer graphene, we find that only stacks with an odd number of layers are quantum spin Hall insulators. However, the size of the gap is the same as that for a monolayer, and thereby most likely is too small to be detected experimentally. In contrast, we propose a toy model for graphene encapsulated between two semiconducting layers with strong SOC and a trivial gap. Our model shows that a nontrivial gap can be opened in graphene whose magnitude is controlled by the atomic spin-orbit coupling of the adjacent layers. Our model provides a qualitative understanding of recent DFT calculations [58] as well as recent experimental work [37] and shows a promising route to observe the quantum spin Hall phase in graphene.

\section{ACKNOWLEDGMENTS}

J.F.R. acknowledges financial supported by MECSpain (FIS2010-21883-C02-01) and Generalitat Valenciana (ACOMP/2010/070), Prometeo. This work has been financially supported in part by FEDER funds. We acknowledge financial support by Marie-Curie-ITN607904-SPINOGRAPH. J.L.L. and N.G. thank the hospitality of the Departamento de Fisica Aplicada at the Universidad de Alicante.
[1] C. L. Kane and E. J. Mele, Phys. Rev. Lett. 95, 226801 (2005).

[2] H. Min, J. E. Hill, N. A. Sinitsyn, B. R. Sahu, L. Kleinman, and A. H. MacDonald, Phys. Rev. B 74, 165310 (2006).

[3] Y. Yao, F. Ye, X. L. Qi, S. C. Zhang, and Z. Fang, Phys. Rev. B 75, 041401(R) (2007).

[4] M. Gmitra, S. Konschuh, C. Ertler, C. Ambrosch-Draxl, and J. Fabian, Phys. Rev. B 80, 235431 (2009).

[5] B. Andrei Bernevig, T. L. Hughes, and S. Zhang, Science 314, 5806 (2006).

[6] M. König, S. Wiedmann, C. Brüne, A. Roth, H. Buhmann, L. W. Molenkamp, X. Qi, and S. Zhang, Science 318, 766 (2007).

[7] C. Sabater, D. Gosálbez-Martínez, J. Fernández-Rossier, J. G. Rodrigo, C. Untiedt, and J. J. Palacios, Phys. Rev. Lett. 110, 176802 (2013).

[8] I. K. Drozdov, A. Alexandradinata, S. Jeon, S. Nadj-Perge, H. Ji, R. J. Cava, B. A. Bernevig, and A. Yazdani, Nat. Phys. 10, 664 (2014)

[9] H. Zhang, Y. Xu, J. Wang, K. Chang, and S. C. Zhang, Phys. Rev. Lett. 112, 216803 (2014).

[10] I. Knez and R. Du, Front. Phys. 7, 200 (2011).

[11] P. Vogt, P. De Padova, C. Quaresima, J. Avila, E. Frantzeskakis, M. C. Asensio, A. Resta, B. Ealet, and G. Le Lay, Phys. Rev. Lett. 108, 155501 (2012).

[12] A. Fleurence, R. Friedlein, T. Ozaki, H. Kawai, Y. Wang, and Y. Yamada-Takamura, Phys. Rev. Lett. 108, 245501 (2012).

[13] C. Xu, G. Luo, Q. Liu, J. Zheng, Z. Zhang, S. Nagase, Z. Gao, and J. Lu, Nanoscale 4, 3111 (2012).

[14] M. Houssa, G. Pourtois, V. V. Afanas'ev, and A. Stesmans, Appl. Phys. Lett. 96, 082111 (2010).

[15] L. Xu, Y. Zhou, and C.-D. Gong, J. Phys.: Condens. Matter 25, 335503 (2013).

[16] S. Murakami, Phys. Rev. Lett. 97, 236805 (2006).
[17] J.-J. Zhou, W. Feng, G.-B. Liu, and Y. Yao, New J. Phys. 17, 015004 (2015).

[18] M. E. Davila, L. Xian, S. Cahangirov, A. Rubio, and G. Le Lay, New J. Phys. 16, 095002 (2014).

[19] B. Lalmi, H. Oughaddou, H. Enriquez, A. Kara, S. Vizzini, B. Ealet, and B. Aufray, Appl. Phys. Lett. 97, 223109 (2010).

[20] C. C. Liu, H. Jiang, and Y. Yao, Phys. Rev. B 84, 195430 (2011).

[21] P. Tang, P. Chen, W. Cao, H. Huang, S. Cahangirov, L. Xian, Y. Xu, S. C. Zhang, W. Duan, and A. Rubio, Phys. Rev. B 90, 121408 (2014).

[22] C. Petit and T. J. Bandosz, Adv. Mater. 21, 4753 (2009).

[23] T. Rodenas, I. Luz, G. Prieto, B. Seoane, H. Miro, A. Corma, F. Kapteijn, F. X. Llabres i Xamena, and J. Gascon, Nat. Mater. 14, 48 (2015).

[24] G. Giovannetti, P. A. Khomyakov, G. Brocks, P. J. Kelly, and J. Van Den Brink, Phys. Rev. B 76, 073103 (2007).

[25] K. Kośmider and J. Fernández-Rossier, Phys. Rev. B 87, 075451 (2013).

[26] A. K. Geim and I. V. Grigorieva, Nature (London) 499, 419 (2013).

[27] E. McCann and M. Koshino, Rep. Prog. Phys. 76, 056503 (2013).

[28] E. McCann and V. I. Falko, Phys. Rev. Lett. 96, 086805 (2006).

[29] S. Konschuh, M. Gmitra, D. Kochan, and J. Fabian, Phys. Rev. B 85, 115423 (2012).

[30] F. Zhang, B. Sahu, H. Min, and A. H. MacDonald, Phys. Rev. B 82, 035409 (2010)

[31] C. Coletti, S. Forti, A. Principi, K. V. Emtsev, A. A. Zakharov, K. M. Daniels, B. K. Daas, M. V. S. Chandrashekhar, T. Ouisse, D. Chaussende, A. H. MacDonald, M. Polini, and U. Starke, Phys. Rev. B 88, 155439 (2013). 
[32] H. Min and A. H. MacDonald, Prog. Theor. Phys. Suppl. 176, 227 (2008).

[33] F. Freitag, J. Trbovic, M. Weiss, and C. Schönenberger, Phys. Rev. Lett. 108, 076602 (2012).

[34] J. Velasco, Jr., Y. Lee, F. Zhang, K. Myhro, D. Tran, M. Deo, D. Smirnov, A. H. MacDonald, and C. N. Lau, Nat. Commun. 5, 4550 (2014).

[35] Y. Lee, D. Tran, K. Myhro, J. Velasco, N. Gillgren, C. N. Lau, Y. Barlas, J. M. Poumirol, D. Smirnov, and F. Guinea, Nat. Commun. 5, 5656 (2014).

[36] A. L. Grushina, D.-K. Ki, M. Koshino, A. A. L. Nicolet, C. Faugeras, E. McCann, M. Potemski, and A. F. Morpurgo, Nat. Commun. 6, 6419 (2015).

[37] A. Avsar, J. Y. Tan, T. Taychatanapat, J. Balakrishnan, G. K. W. Koon, Y. Yeo, J. Lahiri, A. Carvalho, A. S. Rodin, E. C. T. O'Farrell, G. Eda, A. H. Castro Neto, and B. Özyilmaz, Nat. Commun. 5, 4875 (2014).

[38] A. Cortijo, A. G. Grushin, and M. A. H. Vozmediano, Phys. Rev. B 82, 195438 (2010).

[39] F. Guinea, New J. Phys. 12, 083063 (2010).

[40] E. Prada, P. San-Jose, L. Brey, and H. Fertig, Solid State Commun. 151, 1075 (2011).

[41] Z. Qiao, W.-K. Tse, H. Jiang, Y. Yao, and Q. Niu, Phys. Rev. Lett. 107, 256801 (2011).

[42] S. Konschuh, M. Gmitra, and J. Fabian, Phys. Rev. B 82, 245412 (2010).
[43] A. Kormányos and G. Burkard, Phys. Rev. B 87, 045419 (2013).

[44] E. McCann and M. Koshino, Phys. Rev. B 81, 241409 (2010).

[45] S. Fratini, D. Gosalbez-Martinez, P. Merodio Cámara, and J. Fernández-Rossier, Phys. Rev. B 88, 115426 (2013).

[46] J. W. Rhim and K. Moon, Phys. Rev. B 84, 035402 (2011).

[47] W. Norimatsu and M. Kusunoki, Phys. Rev. B 81, 161410 (2010).

[48] J. C. Charlier, X. Gonze, and J. P. Michenaud, Europhys. Lett. 28, 403 (1994).

[49] J. Charlier, X. Gonze, and J. Michenaud, Carbon 32, 289 (1994).

[50] J. C. Slater and G. F. Koster, Phys. Rev. 94, 1498 (1954)

[51] D. Gosalbez-Martinez, J. J. Palacios, and J. Fernández-Rossier, Phys. Rev. B 83, 115436 (2011).

[52] M. I. Katsnelson, Graphene: Carbon in Two Dimensions (Cambridge University Press, Cambridge, UK, 2012).

[53] http://elk.sourceforge.net/.

[54] L. Fu and C. L. Kane, Phys. Rev. B 76, 045302 (2007).

[55] L. Fu and C. L. Kane, Phys. Rev. B 74, 195312 (2006).

[56] T. Fukui, Y. Hatsugai, and H. Suzuki, J. Phys. Soc. Jpn. 74, 1674 (2005).

[57] T. Fukui and Y. Hatsugai, J. Phys. Soc. Jpn. 76, 053702 (2007).

[58] J. Zhang, C. Triola, and E. Rossi, Phys. Rev. Lett. 112, 096802 (2014).

[59] T. P. Kaloni, L. Kou, T. Frauenheim, and U. Schwingenschlögl, Appl. Phys. Lett. 105, 233112 (2014). 